\title{
Positive and negative curvatures nested in antiresonant hollow-core fiber
}

\author{
Md Imran Hasan ${ }^{1, *}$, NAIL Akhmediev ${ }^{1}$, And Wonkeun Chang ${ }^{1}$ \\ ${ }^{1}$ Optical Sciences Group, Research School of Physics and Engineering, The Australian National University, Acton ACT 2601, Australia \\ *Corresponding author: imran.hasan@anu.edu.au
}

Compiled May 27, 2021

We propose a negative-curvature hollow-core fiber that has an elliptical nested element in the antiresonant tubes. The additional elliptical element effectively adds two curvatures, namely a positive and a negative curvature. Our numerical study shows that it enhances the confinement of the light in the core. Moreover, the nested elements provide an extra degree of freedom that can be exploited for suppressing higherorder modes through the change of the ellipticity. The resulting low confinement loss and single-mode guidance properties of the proposed fiber make it a suitable candidate for applications in ultrashort pulse delivery and gas-based nonlinear systems. (๑ 2021 Optical Society of America

OCIS codes: (060.4005) Microstructured fibers; (060.2310) Fiber optics;(060.2280) Fiber design and fabrication.

http://dx.doi.org/10.1364/ao.XX.XXXXXX

We propose a negative-curvature hollow-core fiber that has an elliptical nested element in the antiresonant tubes. The additional elliptical element effectively adds two curvatures, namely a positive and a negative curvature. Our numerical study shows that it enhances the confinement of the light in the core. Moreover, the nested elements provide an extra degree of freedom that can be exploited for suppressing higherorder modes through the change of the ellipticity. The resulting low confinement loss and single-mode guidance properties of the proposed fiber make it a suitable candidate for applications in ultrashort pulse delivery and gas-based nonlinear systems. (๑) 2021 Optical Society of America

OCIS codes: (060.4005) Microstructured fibers; (060.2310) Fiber optics;(060.2280) Fiber design and fabrication.

http://dx.doi.org/10.1364/ao.XX.XXXXXX

Hollow-core (HC) fibers have attracted significant interest amongst the researchers. In HC fibers, the light can be guided with much smaller dispersion and nonlinearity than in solidcore fibers, which could be very useful for delivering ultrashort or high-energy pulses over a long distance [1]. Moreover, they can be filled with gas or liquid, offering an ideal basis for studies of light-matter interactions [2]. This approach allows many applications, such as supercontinuum generations [3, 4], gas-based lasers [5] and high-harmonic generation [6]. In particular, the possibility to easily tune the dispersion and nonlinear properties through changing the filling material density means that they provide a versatile platform for studying ultrafast nonlinear optics [3].

In recent years, there has been a remarkable improvement in the transmission properties of HC fibers, thanks to an assorted range of innovative cladding structure designs that effectively reduces the light leakage in the core. There are a large variety of HC fibers that have been studied so far. This includes simple capillary fibers [7], photonic bandgap fibers [8], kagomélattice photonic crystal fibers [9] and negative curvature (NC) fibers [10-12]. Among these, NC fibers have been shown to have excellent guiding properties, featuring low transmission loss over a broadband of frequencies, small light-glass overlap and small group-velocity dispersion [13]. There have been many research efforts recently to reduce the transmission loss of NC fibers by enhancing the field confinement in the core region. Notably, a significant improvement in the confinement loss could be achieved by introducing variations to the cladding structures, such as including one or more nested elements in the antiresonant tubes [13,14], and using elliptical capillaries [15] or ice-cream cone-shape capillaries as the antiresonant tubes [16]. In this work, we present a new NC fiber design that incorporates an elliptical nested element in the antiresonant tubes. We study, numerically, the effect of adding these additional curvatures on the confinement loss.

Figure 1 shows the proposed NC fiber structure. The core has a diameter $D=30 \mu \mathrm{m}$ and an inner capillary glass web thickness $t=0.4 \mu \mathrm{m}$. These values will remain fixed in the calculations presented below. For a given number of the antiresonant tubes $n$ and the perimeter gap between two antiresonant tubes $g$, the outer diameter of the antiresonant tubes can be obtained using $D_{1}=[g-D \sin (\pi / n)] /[\sin (\pi / n)-1]$. For the proposed fiber, $n=6$ and hence $D_{1}=D-2 g$, i.e. $D_{1}=D$ when $g=0$. This $n=6$ gives the largest possible separation between the core and the outer capillary, while still keeping the antiresonant tube smaller than the core to confine the light well within the core 
[14]. In previous studies, the nested elements in the antiresonant tubes were attached to the outer capillary [12-14]. On the contrary, we use an elliptical tube as the nested element in each antiresonant tube, such that the nested elliptical tube and the antiresonant tube have the same center position as shown in Fig. 1. The circularity of the elliptical tube is defined by $\eta=r_{2} / r_{1}$, where $r_{1}$ and $r_{2}$ are its major and minor axes, respectively. Since the nested element needs to be attached to the antiresonant tube, we set $r_{1}=D_{1}-1.96 t$. This arrangement yields an additional reflecting curvature without increasing the number of nested elements, which further reduces the confinement loss by improving the phase mismatch between the core mode and the cladding modes. Moreover, the ellipticity in the nested elements provides an extra degree of freedom for controlling the guiding properties.

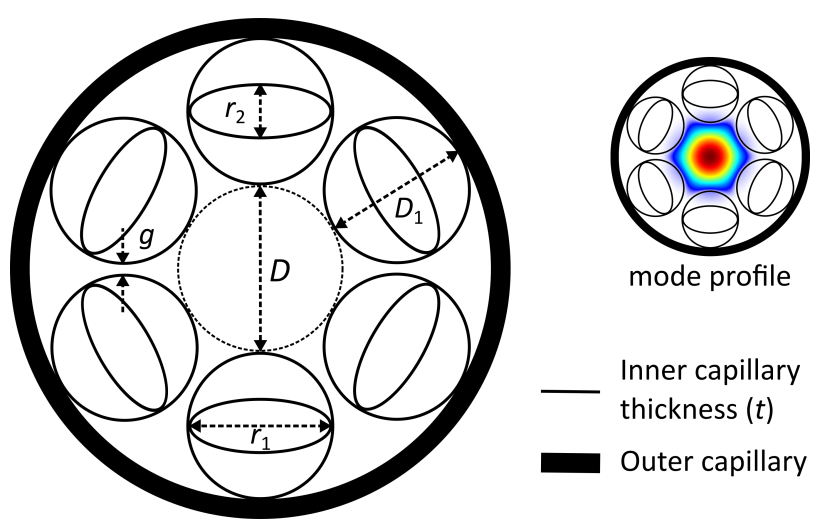

Fig. 1. The geometry of the proposed NC fiber with core diameter $D=30 \mu \mathrm{m}$ and inner glass thickness $t=0.4 \mu \mathrm{m}$. These values are fixed throughout the numerical calculations here. The mode profile at $\lambda=1.06 \mu \mathrm{m}$ is shown in the up-right corner.

It is a challenging task to fabricate the elliptical nested element inside the circular antiresonant tube. One may realize this by following the stack-and-draw procedure for fabricating the cane for a dual-membrane fiber [17]. In order to achieve a desired ellipticity, the pressure needs to be carefully adjusted during the cane drawing process. Six of these canes can then be inserted into a jacket tube to fabricate the final structure.

We use the finite-element method with quadratic finite elements to calculate guidance properties of the fiber for different values of the structural parameters. In order to minimize the computational domain, we use a quarter structure of the fiber. We obtain reliable numerical results by applying an optimized perfectly matched layer on the boundary, as well as using a very high mesh resolution of up to $\lambda / 15$ in the air-core and silica-cladding area, and $\lambda / 8$ in air-cladding area.

First, we study the effect of the perimeter gap $g$ on the confinement loss $L_{c}$. Figure 2 shows $L_{c}$ as a function of wavelength for different values of $g$, while keeping the circularity fixed at $\eta=0.5$. It shows that introducing a small gap improves the confinement loss. The gap increases the curvature of the antiresonant tubes by reducing $D_{1}$. Also, the effective core size increases with the gap, which results in a bigger angle of incidence, and hence an improved reflectivity at the core-cladding interface. These factors enhance the inhibited coupling between the core mode and the cladding modes, and confine better the light in the core. Additionally, by introducing the gap, we can avoid the formation of extra resonators between the antiresonant tubes, which further decreases the confinement loss. On the other hand, if the gap is too big $(g>1 \mu \mathrm{m})$, much of the light will leak through the gap. Furthermore, the distance between the core and the outer cladding becomes too small, which significantly increases the coupling between the core mode and the cladding modes. Therefore, the confinement loss becomes high, if the gap is too big, and there is an optimum perimeter gap where the confinement loss is at its minimum. In our example, this is $g=1 \mu \mathrm{m}$ as shown in Fig. 2 . We use this optimum perimeter gap value in the remaining sections.

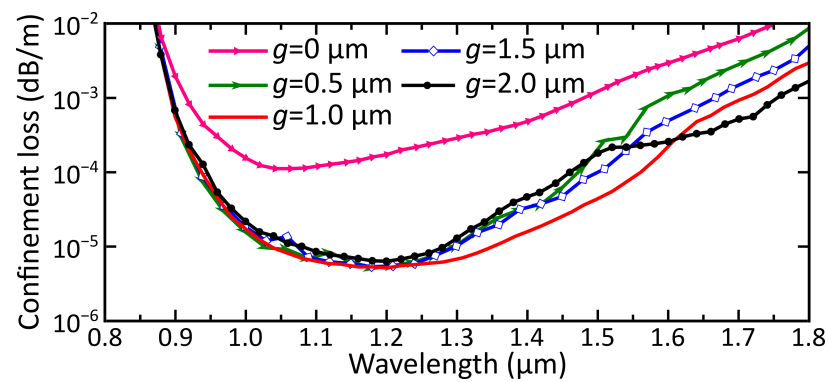

Fig. 2. The confinement loss $L_{c}$ as a function of the wavelength for several different values of $g$ when $\eta=0.5$.

Figure 3 presents $L_{c}$ as a function of the wavelength for several different values of $\eta$. This plot reveals that the loss is greatly reduced when $\eta$ is decreased, especially at wavelengths far from the structural resonance $(\lambda=0.81 \mu \mathrm{m})$. However, the loss does not continue to decrease for $\eta \rightarrow 0$, but increases again for small values of $\eta$ with a minimum loss at $\eta \approx 0.3$. (Note that $\eta=0$ is essentially a single planar sheet of silica nested inside the antiresonant tube.) This is because the gap between the curvatures of the nested element becomes smaller, and they effectively approach a single curvature. Figs. 2 and 3 also confirm that there is a resonance at $\lambda=0.81 \mu \mathrm{m}$ caused by the glass web thickness, which is in a good agreement with the antiresonant reflecting optical waveguide model for the given glass web thickness [18].

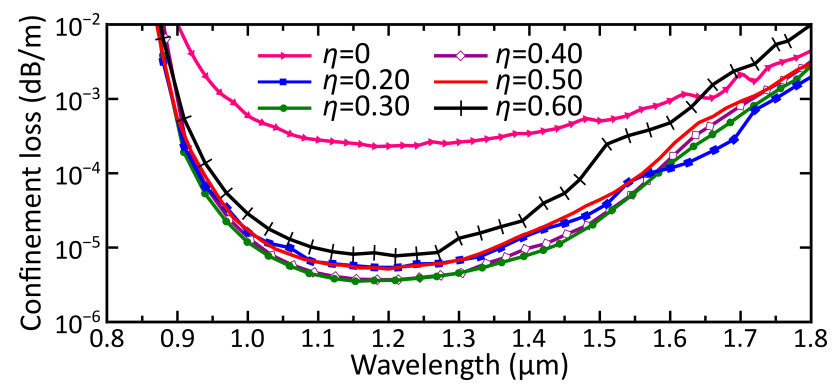

Fig. 3. The confinement loss as a function of the wavelength for several different values of $\eta$ when $g=1 \mu \mathrm{m}$.

In order to study numerically the effect of each curvature in the cladding of the proposed fiber, we successively add each curvature, and calculate the confinement loss as presented in Fig. 4 . This plot shows clearly that increasing the number of curvatures substantially improves the light confinement, regardless of whether the curvature is positive or negative. This happens because the increase of the number of cladding curvatures prevents effectively the coupling between the fundamental core mode and the cladding modes thus enhancing the reflection at 
the core-cladding interface. The relative confinement strength between the negative curvature and the positive curvature can also be compared in Fig. 4. It clearly reveals that the negative curvature confines the light much stronger than the positive curvature, which is in accordance with the previous studies on the effects of negative curvature [10,16, 19]. We also calculated the confinement loss curve for one element nested antiresonant nodeless fiber (NANF) which has already been investigated in several previous studies $[13,14]$. This is presented in Fig. 4 . The comparison shows that the proposed structure has much lower confinement loss across the entire spectral range studied herein.

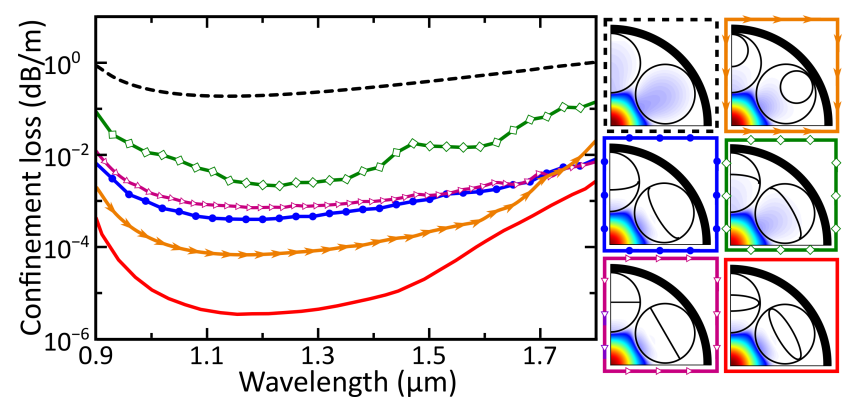

Fig. 4. The effect of the cladding curvatures on the confinement loss when $\eta=0.3$. The fundamental mode profile at $\lambda=1.06 \mu \mathrm{m}$ in each case is shown in the right-hand side panels.

We also investigate the modal properties of the proposed fiber. Figures 5(a) and 5(b) show the wavelength dependent effective index and the confinement loss, respectively, for the fundamental core mode $\left(\mathrm{LP}_{01}\right)$ and the higher-order core modes $\left(\mathrm{LP}_{11}\right.$ and $\left.\mathrm{LP}_{02}\right)$. The profiles of these modes are also shown in the right hand side panels of Fig. 5. The plot shows that the fundamental core mode has higher effective index than the higherorder core modes. A higher mode index results in a higher angle of incidence and a better reflection at the core-cladding interface. Hence, the fundamental core mode has the lowest confinement loss as can be seen from Fig. 5(b). In Fig. 5(c), the higher-order mode extinction ratio (HOMER) is plotted against the wavelength. HOMER is defined as the ratio between the loss of the higher-order mode having the lowest loss and that of the fundamental mode. It gives an indication of the singlemodeness of the given fiber. Across the entire spectral range from $\lambda=0.9 \mu \mathrm{m}$ to $1.48 \mu \mathrm{m}$, HOMER is relatively high $(>30)$, which ensures that the fiber remains single-mode. The peaks shown in Fig. 5(c) come from the peaks in the confinement loss of $\mathrm{LP}_{11}$ mode. At these wavelengths, the $\mathrm{LP}_{11}$ mode is phase matched to the cladding modes, resulting in a higher confinement loss and HOMER.

One can take advantage of this effect, and design the fiber to have a very high HOMER at a specific wavelength. As an example, Fig. 6 shows the effect of changing $\eta$ on the mode index, confinement loss and HOMER for the core modes $\left(\mathrm{LP}_{01}, \mathrm{LP}_{11}\right.$ and $\mathrm{LP}_{02}$ ), as well as six cladding modes at $\lambda=1.06 \mu \mathrm{m}$. This is an important wavelength where high-power $\mathrm{Yb}$ laser operates, and waveguides that have low-loss single-mode guidance at this wavelength are highly desirable.

Figure 6(a) reveals that the change in the effective index of $\mathrm{LP}_{01}$ mode on $\eta$ is almost unnoticeable, because the index depends mostly on the core size. In contrast, the index of the higherorder core modes $\left(\mathrm{LP}_{11}\right.$ and $\left.\mathrm{LP}_{02}\right)$ shown in the second column and the six cladding modes shown in the third and fourth col-

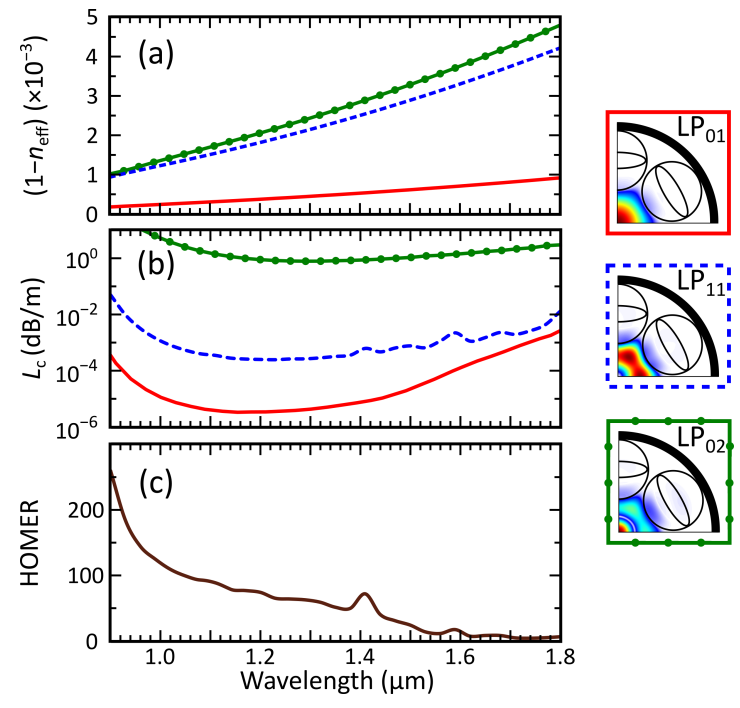

Fig. 5. The wavelength dependence of (a) the effective index, (b) confinement loss of the fundamental mode $\left(\mathrm{LP}_{01}\right)$ and two higher-order modes $\left(\mathrm{LP}_{11}\right.$ and $\left.\mathrm{LP}_{02}\right)$, and (c) HOMER when $\eta=0.3$. The mode profiles are presented on the right-hand side. The box color surrounding the mode profiles inidicates the corresponding core mode curves in (a) and (b).

umn vary with $\eta$. This observation suggests that the phase matching between different modes can be controlled through changing $\eta$. In Fig. 6(a), the phase matching between $\mathrm{LP}_{11}$ mode and each of the first five CMs is achieved at $\eta=0.45,0.43$, $0.45,0.75$ and 0.75 , respectively. Likewise, for the $\mathrm{LP}_{02}$ mode, they occur at $\eta=0.42,0.4,0.42,0.62,0.62$ and 0.8 with all six cladding modes presented. At these phase-matching points, the modes interact through the exchange of energy. Here, the loss increases for the corresponding higher-order core modes, and decreases for the corresponding cladding modes, as shown in Fig. 6(b). On the other hand, $\mathrm{LP}_{01}$ is much less dependent on $\eta$, as there is big index difference between $\mathrm{LP}_{01}$ mode and the cladding modes, and hence a large phase mismatch. Figure 6(c) plots HOMER against $\eta$. The efficient transfer of energy from $\mathrm{LP}_{11}$ mode to the cladding modes through the phase matching at $\eta=0.45,0.75$ implies a high HOMER ( $>1950)$, which indicates an effective suppression of the higher-order modes at $\lambda=1.06 \mu \mathrm{m}$. This shows that the ellipticity of the nested elements can be well-utilized to manage the confinement loss as well as the single-modeness of the proposed fiber.

In order to find the effect of the circularity on the critical bending radius, we plot the loss as a function of the bending radius $R$ at $\lambda=1.06 \mu \mathrm{m}$ for several different values of $\eta$ in Fig. 7(a). Note that $\eta=1$ corresponds to a cladding without any nested element. In all cases where there is a nested element in the cladding tube, the bending loss does not change much for $R>15 \mathrm{~cm}$, but increases rapidly for $R<10 \mathrm{~cm}$. This is because more light in the fundamental mode leaks into the cladding when the bending radius is reduced. This phenomenon is clearly illustrated in Fig. 7(b), where the core mode profile is pushed towards the cladding as $R$ is decreased. It should be noted that, for each $\eta$, there are local peaks at very small bend radii. An example of the $\mathrm{LP}_{01}$ mode profile at the local peak when $\eta=0.55$ is shown in the inset of Fig. 7(a). It reveals that the $\mathrm{LP}_{01}$ mode is strongly phase-matched to the cladding mode at this particular bending radius, increasing the loss further. From 


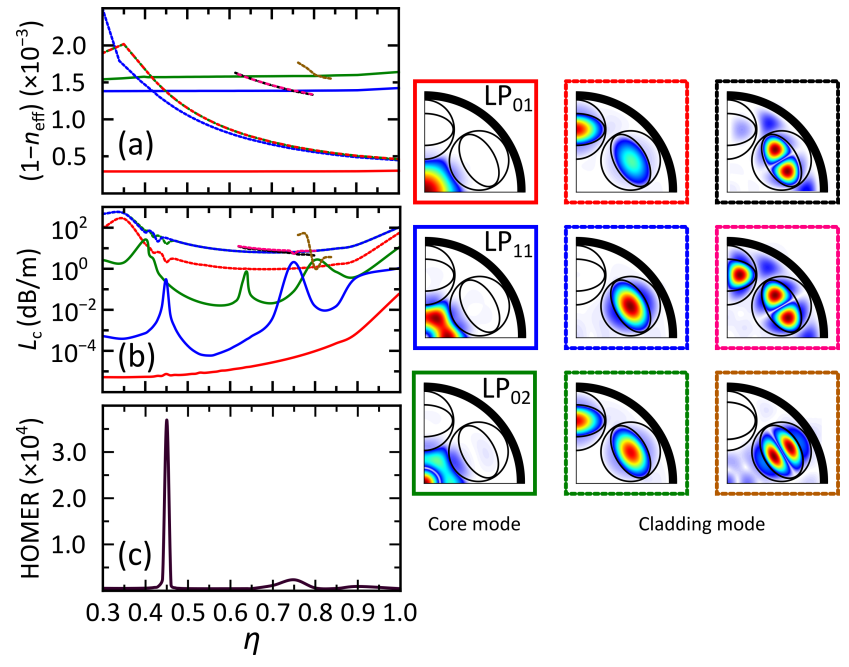

Fig. 6. The circularity dependence of (a) the effective index, (b) confinement loss of the three core modes and six cladding modes, and (c) HOMER at $\lambda=1.06 \mu \mathrm{m}$. The profiles of the core and cladding modes are shown on the second, third and fourth column. The color and the line style of the box surrounding the mode profiles indicate the corresponding core and cladding mode curves in (a) and (b).

Fig. 7(a), we can conclude that the critical bend radius $R_{\text {crit, }}$ which is defined as the radius where the confinement loss is doubled in comparison to that of the straight fiber, increases with the increasing $\eta$, namely, $R_{\text {crit }}=10.5 \mathrm{~cm}, 13 \mathrm{~cm}$ and $15 \mathrm{~cm}$ for $\eta=0.45,0.55$ and 0.65 , respectively. On the other hand, when $\eta=1, R_{\text {crit }}$ is at a much larger value of $23 \mathrm{~cm}$. For a comparison, the bending loss of the existing one-nested-element antiresonant fiber, i.e. NANF structure shown in Fig. 4 , is also presented in Fig. 7(a). Its $R_{\text {crit }}$ is at $13.5 \mathrm{~cm}$.

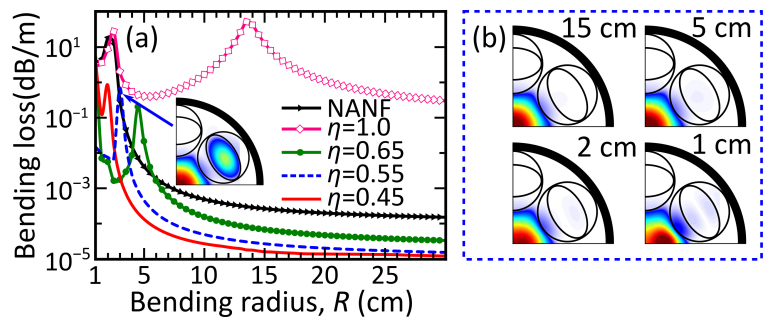

Fig. 7. (a) The bending loss at $\lambda=1.06 \mu \mathrm{m}$ as a function of bending radius $R$ for several different values of $\eta$, and (b) the fundamental mode profile at different bending radii for $\eta=$ 0.55. The inset in (a) shows the mode profile at the local peak.

We also investigated the influence of rotating the antiresonant tubes on the confinement loss. Close up examples of the antiresonant tube rotations are given in Fig. 8: (a) for several different rotation angles $\theta$; and (b) for two different values of $\eta$ superimposed, when $\theta$ is fixed. The rotation may occur unintentionally during the fabrication process, and hence this study gives an indication of the fiber fabrication tolerance. When the tubes are rotated, the symmetry is broken, and we cannot calculate the confinement loss only with the quarter structure. Therefore, here we must use the full structure in the calculation domain. Figure 8(c) shows the confinement loss as a function of the antiresonant tube rotation angle $\theta$ for several different values of $\eta$ at $\lambda=1.06 \mu \mathrm{m}$. It clearly indicates that the confinement loss depends strongly on $\theta$. The loss increases dramatically above a critical angle. One can see from Fig. 8(a), that when the antiresonant element rotates, the extra resonator (ER) formed at the node between the antiresonant tube and its nested element moves closer to the core. This enhances coupling between the core and the cladding modes, and thus the overall loss increases. Figure 8 (c) also shows that the critical angle $\theta_{\text {crit }}$, which is the angle where the confinement loss is doubled from that at $\theta=0$, varies with $\eta ; \theta_{\text {crit }}$ decreases as $\eta$ is reduced, limiting the rotational tolerance. We can explain this by noting the relative position between the negative curvature in the nested element and the extra resonator shown in Fig. 8(b). It shows that when $\eta$ is small, the extra resonator is more easily exposed to the $\mathrm{LP}_{01}$ mode than when $\eta$ is large. Hence, when $\eta$ is small, $\mathrm{LP}_{01}$ mode is significantly affected by the extra resonator at a smaller $\theta$, leading to a small $\theta_{\text {crit }}$. The effect of antiresonant tube rotation was also studied for the exiting one-nested-element antiresonant fiber [13]. Comparing the two results shows that the fiber structure proposed in this work is more susceptible to the rotation due to ER approaching closer to the core with the rotation.

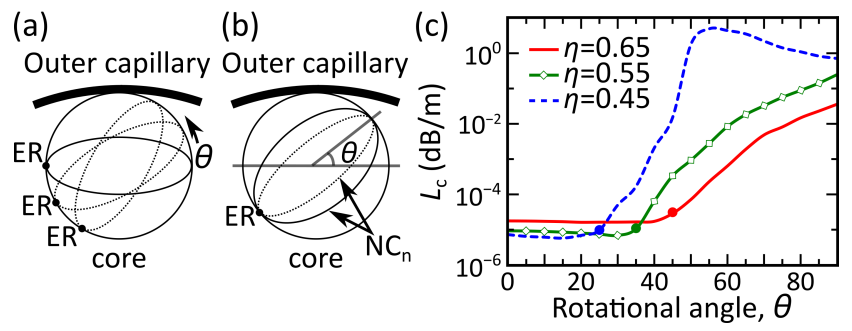

Fig. 8. Schematic diagram of one antiresonant tube in the fiber showing (a) the rotation of the nested element at several different rotation angles, and (b) the rotated nested element for two different $\eta$, when $\theta$ is fixed. ER denotes the extra resonator formed at the node between the antiresonant tube and the nested element. $\mathrm{NC}_{\mathrm{n}}$ is the negative curvature of the nested element. The confinement loss as a function of the rotation angle $\theta$ is presented in (c) for several different values of $\eta$. The solid dots indicate the critical angle $\theta_{\text {crit }}$.

In conclusion, we propose a new type of NC fiber. It has antiresonant tubes nested by an elliptical element that provides active negative and positive curvatures. Our numerical results show that despite using only a single nested element, ultra-low loss is achievable over $\lambda=0.9 \mu \mathrm{m}$ to $1.8 \mu \mathrm{m}$. We also studied the single-modeness of the fiber, which can be effectively controlled by changing the ellipticity of the nested element. Therefore, the fiber is highly suitable for low-loss, single-mode delivery of ultrashort pulses in the near-infrared region, which makes it an excellent candidate for applications in ultrafast nonlinear optics and high-speed data transmissions.

Australian Research Council (ARC) (DE130101432, DP140100265, DP150102057). 


\section{FULL REFERENCES}

1. Y. Y. Wang, X. Peng, M. Alharbi, C. F. Dutin, T. D. Bradley, F. Gérôme, M. Mielke, T. Booth, and F. Benabid, "Design and fabrication of hollowcore photonic crystal fibers for high-power ultrashort pulse transportation and pulse compression," Opt. Lett. 37, 3111-3113 (2012).

2. P. S. J. Russell, P. Holzer, W. Chang, A. Abdolvand, and J. C. Travers, "Hollow-core photonic crystal fibres for gas-based nonlinear optics," Nature Photon. 8, 278-286 (2014). Review.

3. J. C. Travers, W. Chang, J. Nold, N. Y. Joly, and P. S. J. Russell, "Ultrafast nonlinear optics in gas-filled hollow-core photonic crystal fibers," J. Opt. Soc. Am. B 28, A11-A26 (2011).

4. M. I. Hasan, N. Akhmediev, and W. Chang, "Mid-infrared supercontinuum generation in supercritical xenon-filled hollow-core negative curvature fibers," Opt. Lett. 41, 5122-5125 (2016).

5. A. V. V. Nampoothiri, A. Ratanavis, N. Campbell, and W. Rudolph, "Molecular $\mathrm{C}_{2} \mathrm{H}_{2}$ and $\mathrm{HCN}$ lasers pumped by an optical parametric oscillator in the 1.5- $\mu \mathrm{m}$ band," Opt. Express 18, 1946-1951 (2010).

6. O. H. Heckl, C. R. E. Baer, C. Kränkel, S. V. Marchese, F. Schapper, M. Holler, T. Südmeyer, J. S. Robinson, J. W. G. Tisch, F. Couny, P. Light, F. Benabid, and U. Keller, "High harmonic generation in a gas-filled hollow-core photonic crystal fiber," 97, 369-373 (2009).

7. E. A. J. Marcatili and R. A. Schmeltzer, "Hollow metallic and dielectric waveguides for long distance optical transmission and lasers," Bell Syst. Tech. J. 43, 1783-1809 (1964).

8. R. F. Cregan, B. J. Mangan, J. C. Knight, T. A. Birks, P. S. J. Russell, P. J. Roberts, and D. C. Allan, "Single-mode photonic band gap guidance of light in air," Science 285, 1537-1539 (1999).

9. F. Couny, F. Benabid, and P. S. Light, "Large-pitch kagome-structured hollow-core photonic crystal fiber," Opt. Lett. 31, 3574-3576 (2006).

10. A. D. Pryamikov, A. S. Biriukov, A. F. Kosolapov, V. G. Plotnichenko, S. L. Semjonov, and E. M. Dianov, "Demonstration of a waveguide regime for a silica hollow - core microstructured optical fiber with a negative curvature of the core boundary in the spectral region $>3.5$ $\mu \mathrm{m}$," Opt. Express 19, 1441-1448 (2011).

11. W. Belardi and J. C. Knight, "Hollow antiresonant fibers with reduced attenuation," Opt. Lett. 39, 1853-1856 (2014).

12. F. Yu and J. Knight, "Negative curvature hollow core optical fiber," IEEE J. Sel. Top. Quant. 22, 1-11 (2016).

13. W. Belardi, "Design and properties of hollow antiresonant fibers for the visible and near infrared spectral range," J. Lightwave Technol. 33, 4497-4503 (2015).

14. F. Poletti, "Nested antiresonant nodeless hollow core fiber," Opt. Express 22, 23807-23828 (2014).

15. M. S. Habib, O. Bang, and M. Bache, "Low-loss single-mode hollowcore fiber with anisotropic anti-resonant elements," Opt. Express 24, 8429-8436 (2016).

16. W. Belardi and J. C. Knight, "Effect of core boundary curvature on the confinement losses of hollow antiresonant fibers," Opt. Express 21, 21912-21917 (2013).

17. J. Shi, X. Feng, Z. Lian, N. White, W. H. Loh, F. Poletti, and P. Horak, "Fabrication of multiple parallel suspended-core optical fibers by sheetstacking," Opt. Fiber Technol. 20, 395 - 402 (2014).

18. M. A. Duguay, Y. Kokubun, T. L. Koch, and L. Pfeiffer, "Antiresonant reflecting optical waveguides in $\mathrm{SiO}_{2}$-Si multilayer structures," Appl. Phys. Lett. 49, 13-15 (1986).

19. Y. Y. Wang, N. V. Wheeler, F. Couny, P. J. Roberts, and F. Benabid, "Low loss broadband transmission in hypocycloid-core kagome hollow-core photonic crystal fiber," Opt. Lett. 36, 669-671 (2011). 